\title{
Erratum to: Rate of Convergence in Trotter's Approximation Theorem
}

\section{Michele Campiti · Cristian Tacelli}

Published online: 6 March 2010

(C) Springer Science+Business Media, LLC 2010

\section{Erratum to: Constr Approx (2008) 28: 333-341 \\ DOI 10.1007/s00365-008-9017-z}

In this note we discuss the validity of Theorem 1.1 stated in [1] and give a correct version of formula $[1,(1.4)]$ (Statement 1).

Moreover, we also give some cases in which formula [1, (1.4)] holds with further assumptions (Statement 2).

Finally, we make some clarifications in order to justify the validity of the results concerning the application to Bernstein operators given in [1, Sect. 2] (Statement 3).

Statement 1 Formula [1, (1.4)] becomes

$$
\begin{aligned}
\left\|T(t) u-L_{n}^{k(n)} u\right\| \leq & M e^{\omega t e^{\omega / n}} \int_{0}^{t} e^{-s \omega e^{\omega / n}} \psi_{n}(T(s) u) d s \\
& +M \exp \left(\omega t_{n} e^{\omega / n}\right)\left|\frac{k(n)}{n}-t\right| \varphi_{n}(u) \\
& +M \exp \left(2 \omega \frac{k(n)}{n} e^{\omega / n}\right)\left(\frac{\omega}{n} \frac{k(n)}{n}+\frac{\sqrt{k(n)}}{n}\right) \varphi_{n}(u)
\end{aligned}
$$

for every $u \in D \cap\{v \in D \mid T(t) v \in D\}$.

The online version of the original article can be found under doi:10.1007/s00365-008-9017-z.

M. Campiti $(\bowtie) \cdot$ C. Tacelli

Department of Mathematics, University of Salento, P.O. Box 193, 73100 Lecce, Italy

e-mail: michele.campiti@unisalento.it

C. Tacelli

e-mail: cristian.tacelli@unisalento.it 
Indeed, the term $\left\|T(t) u-S_{n}(t)\right\|$ at the right-hand side of [1, (1.6)] satisfies

$$
\begin{aligned}
T(t) u-S_{n}(t) u & =\int_{0}^{t} \frac{d}{d t} S_{n}(t-s) T(s) u d s \\
& =\int_{0}^{t} S_{n}(t-s)\left(A-A_{n}\right) T(s) u d s,
\end{aligned}
$$

and from $[1,(1.5)]$ and the inequality $e^{x}-1 \leq x e^{x}, x \geq 0$, we have

$$
\begin{aligned}
\left\|T(t) u-S_{n}(t)\right\| & \leq M \int_{0}^{t} e^{n(t-s)\left(e^{\omega / n}-1\right)}\left\|\left(A-A_{n}\right) T(s) u\right\| d s \\
& \leq M \int_{0}^{t} e^{\omega(t-s) e^{\omega / n}}\left\|\left(A-A_{n}\right) T(s) u\right\| d s \\
& =M e^{\omega t e^{\omega / n}} \int_{0}^{t} e^{-s \omega e^{\omega / n}}\left\|\left(A-A_{n}\right) T(s) u\right\| d s \\
& \leq M e^{\omega t e^{\omega / n}} \int_{0}^{t} e^{-s \omega e^{\omega / n}} \psi_{n}(T(s) u) d s .
\end{aligned}
$$

Statement 2 Formula [1, (1.4)] is valid if we add one of the following assumptions in [1, Theorem 1.1]:

1. The operators $L_{n}$ commute each other, i.e., $L_{n} L_{m}=L_{m} L_{n}$ for every $n, m \geq 1$.

In this case the proof requires no modification since the estimate of the first term given after (1.6) holds true (see, e.g., [2, p. 215]).

2. We have

$$
\left\|\left(A_{n}-A\right) T(t) u\right\| \leq\|T(t)\| \psi_{n}(u)
$$

for every $u \in D$.

In this case from (2) we have

$$
\begin{aligned}
\left\|T(t) u-S_{n}(t)\right\| & \leq M e^{\omega t e^{\omega / n}} \int_{0}^{t} e^{-s \omega e^{\omega / n}}\|T(s)\| \psi_{n}(u) d s \\
& \leq M^{2} \psi_{n}(u) e^{\omega t e^{\omega / n}} \int_{0}^{t} e^{-s \omega e^{\omega / n}} e^{\omega s} d s \\
& \leq M^{2} t \psi_{n}(u) e^{\omega t e^{\omega / n}} .
\end{aligned}
$$

We observe that condition (3) holds if:

(i) the operators $L_{n}$ commute with the limit semigroup $T(t)$, i.e., $L_{n} T(t)=$ $T(t) L_{n}$ for every $n \in \mathbb{N}$ and $t \geq 0$;

(ii) $T(t)(D) \subset D$ for every $t \geq 0$, and

$$
\left\|\psi_{n}(T(t) u)\right\| \leq\|T(t)\| \psi_{n}(u)
$$

for every $u \in D$. 
Statement 3 We point out that the application to the Bernstein operators is valid as well, since the above assumption (ii) is satisfied. Indeed, we observe that the semigroup generated by the closure of the differential operator (2.1) maps $C^{m}\left(K_{d}\right)$ into $C^{m}\left(K_{d}\right)$ for every $m \geq 1$, and, moreover (see [3]),

$$
\|T(t) f\|_{C^{m}\left(K_{d}\right)} \leq\|f\|_{C^{m}\left(K_{d}\right)} .
$$

From the classical interpolation theory, it is well known that the existence of a linear extension operator which continuously maps $C^{2}\left(K_{d}\right)$ into $C^{2}\left(\mathbb{R}^{d}\right), C^{3}\left(K_{d}\right)$ into $C^{3}\left(\mathbb{R}^{d}\right)$, and $C^{2+\alpha}\left(K_{d}\right)$ into $C^{2+\alpha}\left(\mathbb{R}^{d}\right)$ for every $\left.\alpha \in\right] 0$, $1\left[\right.$ yields that $C^{2, \alpha}\left(K_{d}\right)$ is an intermediate space between $C^{2}\left(K_{d}\right)$ and $C^{3}\left(K_{d}\right)$.

The existence of a continuous linear operator $E$ from $C^{m}\left(K_{d}\right)$ to $C^{m}\left(\mathbb{R}^{d}\right)$, from $C^{m+\alpha}\left(K_{d}\right)$ to $C^{m+\alpha}\left(\mathbb{R}^{d}\right)$, and from $C^{m+1}\left(K_{d}\right)$ to $C^{m+1}\left(\mathbb{R}^{d}\right)$ may be easily obtained similarly to the proof of [4, Lemma 6.37].

First of all, we observe that the construction of an extension operator can be reduced to the case where the domain is a quadrant $Q_{k}=\left\{x \in \mathbb{R}^{d} \mid x_{1}, x_{2}, \ldots, x_{k} \geq 0\right\}$. Indeed, it is possible to use a partition of unity and suitable local coordinates near the boundary which maps $U \cap K_{d}$ into $V \cap Q_{k}$ where $U$ and $V$ are neighborhoods of $\partial K_{d}$ and $\partial Q_{k}$. For every $x \in \partial K_{d}$ the existence of these local coordinates is achieved by considering the following cases. If $0 \leq x_{1}+\cdots+x_{d}<1$ and $x_{i}=0$ for $i \in J$ where $J$ is a nonempty subset of $\{1,2, \ldots, d\}$, then we can consider a linear invertible map $T$ which permutes the coordinates with indices in $J$ into the coordinates $\left\{x_{1}, \ldots, x_{|J|}\right\}$. Then there exists a neighborhood $U$ of $x$ such that $T\left(U \cup K_{d}\right)=U \cup Q_{|J|}$.

If $x_{1}+\cdots+x_{d}=1$, there exists at least one $i \in\{1,2, \ldots, d\}$ for which $x_{i}>0$, and we can consider the linear map $\left(x_{1}, \ldots, x_{d}\right) \mapsto\left(x_{1}^{\prime}=x_{1}, \ldots, x_{i}^{\prime}=1-x_{1}-x_{2}-\cdots-\right.$ $\left.x_{d}, \ldots, x_{d}^{\prime}=x_{d}\right)$; hence we reduce to the preceding case since $x_{1}^{\prime}+x_{2}^{\prime}+\cdots+x_{d}^{\prime}=$ $1-x_{i}<1$.

The second step consists in defining the extension operator over $Q_{k}$. This is achieved using the Hestenes-Whitney extension method. First we consider the domain $\mathbb{R}_{r+}^{d}=\left\{x \in \mathbb{R}^{d} \mid x_{r} \geq 0\right\}$ and define the extension operator $\Pi_{r}: C\left(\mathbb{R}_{r+}^{d}\right) \rightarrow$ $C\left(\mathbb{R}_{r+}^{d}\right)$ by setting

$$
\Pi_{r} f(x)= \begin{cases}f\left(x_{1}, \ldots, x_{n}\right) & \text { if } x_{r} \geq 0 \\ \sum_{k=1}^{m+1} \lambda_{k} f\left(x_{1}, \ldots,-k x_{r}, \ldots, x_{n}\right) & \text { if } x_{r} \leq 0\end{cases}
$$

where $\lambda_{k}, k=1, \ldots, m+1$ are constants determined by the system of equation

$$
\sum_{k=1}^{m+1}(-k)^{i} \lambda_{k}=1, \quad i=0,1, \ldots, m .
$$

If $f \in C^{m+1}\left(\mathbb{R}_{r+}^{d}\right)$, the function $\Pi_{r} f$ has continuous derivatives up to the order $m+1$, and

$$
\left\|\Pi_{r} f\right\|_{C^{m+1}\left(\mathbb{R}^{d}\right)} \leq C\|f\|_{C^{m+1}\left(\mathbb{R}_{r+}^{d}\right)} ;
$$

moreover, it is also easy to check that $\Pi_{r} f \in C^{m}\left(\mathbb{R}^{d}\right)$ if $f \in C^{m}\left(\mathbb{R}_{r+}^{d}\right)$ and $\Pi_{r} f \in$ $C^{m+\alpha}\left(\mathbb{R}^{d}\right)$ if $f \in C^{m+\alpha}\left(\mathbb{R}_{r+}^{d}\right)$ for every $0<\alpha<1$. Now the extension operator for $Q_{k}$ is obtained by considering the operator $\Pi=\Pi_{k} \circ \Pi_{k-1} \circ \cdots \circ \Pi_{1}$. 
Returning to our situation, $C^{2, \alpha}\left(K_{d}\right)$ is an intermediate space between $C^{2}\left(K_{d}\right)$ and $C^{3}\left(K_{d}\right)$, and therefore $T(t)$ maps $C^{2, \alpha}\left(K_{d}\right)$ into itself and $\|T(t) f\|_{C^{2+\alpha}\left(K_{d}\right)} \leq$ $\|f\|_{C^{2+\alpha}\left(K_{d}\right)}, 0<\alpha<1$. Then

$$
\left\|\left(n\left(B_{n}-I\right)-A\right) T(t) f\right\| \leq \frac{L_{(T(t) f)^{\prime \prime}}}{n^{\alpha / 2}} \leq \frac{L_{f^{\prime \prime}}}{n^{\alpha / 2}},
$$

and consequently the seminorm $\psi$ defined after [1, (2.3), p. 340] satisfies $\psi(T(t) u) \leq$ $\psi(u)$, and estimate $[1,(1.4)]$ holds.

Acknowledgement We are grateful to Dr. Björn Böttcher for calling our attention to the question.

\section{References}

1. Campiti, M., Tacelli, C.: Rate of convergence in Trotter's approximation theorem. Constr. Approx. 28(3), 333-341 (2008)

2. Engel, K.J., Nagel, R.: One-Parameter Semigroups for Linear Evolution Equations. Graduate Texts in Mathematics, vol. 194. Springer, New York (2000)

3. Ethier, S.N.: A class of degenerate diffusion processes occurring in population genetics. Commun. Pure Appl. Math. 29, 483-493 (1976)

4. Gilbarg, D., Trudinger, N.S.: Elliptic Partial Differential Equations of Second Order. Grundlehren der Mathematischen Wissenschaften, vol. 224. Springer, Berlin (1983) 